\title{
The Four-Seasonal Movement of Life
}

\author{
Yan Jinzhong \\ Changsha Jiage'er Machinery Manufacture Co., Ltd., Pingtang Town, Changsha City 410208, Hunan, China
}

Received: March 9, 2015 / Accepted: March 21, 2015 / Published: March 31, 2015.

\begin{abstract}
The four-seasonal movement is the general movement of the universe. Galaxies, living beings, society, man-made worlds and all substances have or take part in the four-seasonal movement. There are transverse four-seasonal movement and longitudinal four-seasonal movement. The former is the breathing movement. All substances have the function of breathing. Breathing involves living beings, celestial bodies, society and man-made systems and living beings of different levels have different ways of breathing. The longitudinal four-seasonal movement of living beings is controlled by genes. The four-seasonal movement is confined. Life originates in seeds and seeds are generated in the four-seasonal movement. Thoughts, ideas, designs and methods are the seeds of celestial bodies, society and man-made worlds. Just like living beings, galaxies, and society also have reproductive function. Reproduction has three major means: self-generation, generation by augmentation and generation by transformation. The reproduction of living beings is self-generation while the reproduction of the solar system and other galaxies is generation by augmentation. Man-made objects, society and galaxies are formed with automatic organization of thoughts, thus called generation by transformation. The solar system and the earth have reproduction function. Living beings began with the combined action of the earth, the solar system and the universe. Life forms of the universe include dominant life and recessive life. Different forms of life transform continuously.
\end{abstract}

Key words: Life, four-seasonal movement, seeds, breathing, reproduction.

\section{Introduction}

The four-seasonal movement and thought movement are the vital movements of the universe [1, 2]. The law of four-seasons is the most important law of the universe; everything in the universe is controlled by this law [1]. Ancient people did more research into the four-seasonal movement than people now because mastering the laws of seasons and forecasting the changes in seasons were of crucial importance to the agriculture then. The Chinese calendar system accurately shows and uses various four-seasonal movements of the solar system.

Although humans all know the four-seasonal movement, modern science does little research into it and few people probe into the big secret hidden behind it. This secret is just the secret of life and the universe.

Corresponding author: Yan Jinzhong, senior engineer, research field: disciplinary sciences. E-mail: yjzh272@tom.com.
To explore the secret of life and the universe, let the author start with the four-seasonal movement!

\section{Phenomena of the Four-Seasonal Movement}

Just as the author mentioned above, humans all know the four-seasonal movement. In spring, the sun shines and everything rouses up from sleeping; in summer, it is the hottest season and plants grow thick; in autumn, crops are ripe and are ready for harvest and the temperature begins to drop; in winter, it is the coldest season and everything goes to sleep. Here comes another four-seasonal movement. In fact, the four-seasonal movement is the dominating movement of the universe; everything in it is on the four-seasonal movement.

\subsection{The Four-Seasonal Movement of Celestial Bodies}

The earth moves around the sun, which is a four-seasonal movement familiar to humans. Likewise, 
the movement of the moon around the earth is also a four-seasonal movement. The rotation of the earth can also be seen as the changes in four seasons [5]. The rotations of the solar system, the Milky Way Galaxy, clusters of galaxies and super clusters are also four-seasonal movements. It is the transverse four-seasonal movement inside the celestial bodies, which includes four-seasonal processes of substance, energy and information absorbing, transforming, using and exporting. The forming, growing, maturing, bursting and dying of celestial bodies in the universe are their longitudinal a four-seasonal movement.

\subsection{The Four-Seasonal Movement of Living Things}

Transversely, living beings' absorbing, transforming, using, storing and exporting substances, energy and information is a four-seasonal movement inside their bodies. Longitudinally, living beings' being born, growing, maturing, declining and dying is the four-seasonal development.

\subsection{The Four-Seasonal Movement of Society}

Transversely, society's absorbing, transforming, using and exporting substances, energy and information is a four-seasonal movement. Longitudinally, dynasty's rising, expanding, decaying and dying is the four-seasonal movement of society. Economy's reviving, prospering, declining and adjusting is also four-seasonal movement.

\subsection{The Four-Seasonal Movement of Man-Made Machines and Systems}

Man-made machines, systems and production lines have four-seasonal structures, at least partially [6]. They absorb, transform, use and export substances, energy and information, which is also a four-seasonal movement. The evolution of the man-made worlds depends on scientific development caused by human thoughts.

\subsection{The Four-Seasonal Movement of Substances}

Classified by four-seasonal functions, all substances are living substances [6]. They have the ability and tendency to actively or passively combine with other substances into a four-seasonal entirety; they all have the four-seasonal movement ability.

\section{Features of the Four-Seasonal Movement}

The features of the four-seasonal movement can be found according to its phenomena.

\subsection{The Transverse Four-Seasonal Movement is the Breathing Movement}

3.1.1 The Movement of Celestial Bodies Around Its Center is a Breathing Movement

The track of this movement is an ellipse. For example, the orbit of the earth around the sun is an ellipse. When the sun-earth system breathes out, the earth moves from the perihelion to the aphelion, and the earth experiences spring and then summer. When the sun-earth system breathes in, the earth moves from the aphelion to the perihelion, and the earth experiences autumn and then winter. The orbit of the moon around the earth is also an ellipse. The sun itself expands and shrinks periodically [7], which is also a breathing movement. The rotation of the earth is also a breathing movement because the earth is an ellipse too.

3.1.2 The Four-Seasonal Movement of Living Beings is a Breathing Movement

Transversely, living body's absorbing, transforming, using and exporting substances, energy and information is a four-seasonal movement inside the living bodies. This process includes absorbing and exporting, which is a breathing movement. Breathing not only means air absorbing and exhaling, but also substances, energy and information absorbing and exhaling. All objects that breathe automatically can be considered as living beings. The four-seasonal structure of breathing is shown in Fig. 1.

\subsubsection{The Breathing Function of Substances}

As to substances, if one substance can repel another, it has the function of breathing out; if the substance 


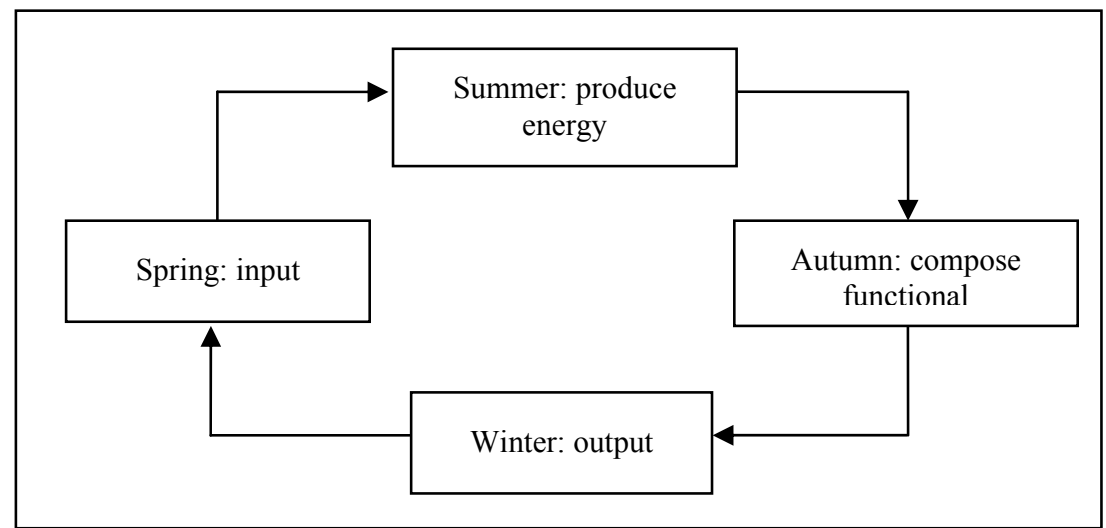

Fig. 1 The four-seasonal structure of breathing.

can attract another, it has the function of breathing in. In fact, all substances in the universe have the function of breathing out or the function of breathing in or both. This function is got because all substances have forces. Substance forces include repulsive force and attractive force. The former is breathing out and the latter is breathing in, such as bond force substances decomposing and composing, heat release and heat absorption, strong interaction inverse fusion and fusion of pi meson exchange, color confinement repelling and attracting, weak interaction expanding and shrinking, electromagnetism interaction repelling and attracting, life structural force gravitational repulsion and universal gravitation [2]. The breathing function of substances forms the basis for life breathing.

\subsection{The Longitudinal Four-Seasonal Development of} Living Beings Is Controlled by Genes

Longitudinally, the being born, growing, maturing, decaying and dying (transforming) of all living beings are four-seasonal movements or the four-seasonal evolution of living beings. Different lives have different four-seasonal life cycles. This is because different lives originate in different seeds and have different genes. The four-seasonal evolution of living beings is controlled by genes.

\subsection{The Four-Seasonal Movement Is Confined}

Breathing is confined. Breathing in and breathing out exist side by side. The confinement of breathing leads to the confinement of four-seasonal movement, namely, the continuous movement of spring, summer, autumn and winter. The four seasons whole [1] must be a complete four-seasonal structure, none is dispensable. Once one season of a four-seasonal living being is missing, it will restore and supply automatically. Thus living beings have the function of automatic recovery and automatic treatment.

\subsection{Life Produces Seeds and Reproduces in the} Four-Seasonal Movement

As is known to all, living beings develop from seeds and produce seeds in the four-seasonal growing process [8]. New seeds become the start of new life development. It is just this continuous four-seasonal circling movement that makes living beings last. Chickens produces eggs and eggs develop into chickens. Chickens and eggs are the two opposite links in living being's four-seasonal movement and are the causes and effects of each other. Reproduction is another important function of living beings and an important node of the four-seasonal movement.

\subsection{The Four-Seasonal Movement Depends on the Surroundings}

When living beings are on their own four-seasonal movement, they participate in that of the surroundings too. The four-seasonal movement of the surroundings 
can be divided into many levels, from small to large. In living nature, single living beings are on their own four-seasonal movement and participate in that of other living beings of their kind and further in that of the regional ecosystem, and then that of the whole earth ecosystem. It is the same case with galaxies, society and man-made systems.

The universe is a living being entirety [1]. The four-seasonal movement is the nature of living beings. The four-seasonal structure is the basic structure of living beings. Naturally, life can be defined as a four-seasonal whole [1].

\section{The Theory of Seeds}

\subsection{Seeds of Living Beings}

Living beings originate in seeds and produce seeds in the four-seasonal development process [8]. Seeds are four-seasonal entireties and have four-seasonal structures. They can automatically combine with the surroundings to go on the four-seasonal movement and development. Seeds are both the original state and the result of living beings.

\subsection{Seeds in the Broad Sense and Their Features}

In fact, no entities (celestial bodies, living beings, society and man-made objects) occur at random. They are expressions of thoughts, ideas, regulations, designs and methods, etc. These thoughts, ideas, regulations, designs and methods are the basic patterns that can organize to form entities automatically [9]. These thoughts, ideas, consciousness, regulations, designs and methods are the original states of things, namely the seeds of things.

Thus, seeds have the following features: (1) Seeds are four-seasonal life bodies or thoughts, ideas, regulations, designs and methods; (2) Seeds have the capacity of automatic four-seasonal breathing movement, the ability of four-seasonal development and the ability to combine with the surroundings; (3) Seeds have an internal automatic dynamical system.

\subsection{Seeds of Various Entities}

\subsubsection{Man-Made Seeds}

The structures, regulations, natures and functions of all substances, objects and celestial bodies can be recognized and described as science, technology, ideas and methods. These science, technology, ideas and methods can be transformed into man-made objects, namely seeds. The bigger and more complex the human cognitive system is, the more complex the seeds are. The bigger the ideas are, the more complex the man-made objects are.

\subsubsection{Seeds of Galaxies in the Universe}

The universe has thoughts. Thoughts are the seeds of the celestial bodies in the universe. The formation of galaxies is the result of automatic organization of the universe thoughts [2]. Seed of different thoughts produce different celestial bodies in the universe.

\subsubsection{Seeds of Living Beings}

Seeds of living beings come from the universe and are the reflection of the life of celestial bodies in the universe. The life of the universe is shown as living beings in the earth's living system.

\subsubsection{Seeds of Society}

All ideas of humans - survival, theology, science, society, economy, and aesthetics, etc.-are the guiding principles or seeds to guide humans to construct society and conduct social movements. These ideas are the results of human thought movement and the outcome of humans knowing of themselves and the natural surroundings.

\section{Breathing}

\subsection{Breathing}

Breathing is a process of an object to exchange energy, substance and information with the external world. It includes energy, substance and information absorbing, digesting, transforming, internal transporting and using and the breathing out (discharge) of internal new substances. 


\subsection{Kinds of Breathing}

\subsubsection{Breathing of living beings}

Living beings exchange substances, energy and information with the surroundings all the time. Through breathing, living beings absorb substances, energy and information from the surroundings, then process, transport and use them inside their bodies, and release the substances, energy and information from their bodies.

\subsubsection{Breathing of Celestial Bodies}

Breathing of celestial bodies also includes substance (gas, liquid, solid) breathing, information breathing and energy breathing. Celestial bodies have all kinds of life features: structure, substances, energy, information, operation, change and thinking, etc. By breathing out, celestial bodies release all their own features. By breathing in, they accept substances, energy and information from the surroundings, process them with their own systems and change them into their own new substances.

\subsubsection{Breathing of Society}

It refers to humans learning from nature, society to gain new ideas, rules, and knowledge, and develop and produce new spiritual and material products to construct society and the artificial world, improve themselves and their living standards.

\subsubsection{Breathing of Man-Made Systems}

Take computer breathing for example: breathing in refers to data, information inputting and processing while breathing out refers to processed data, information storing and outputting. This is just the case with the breathing of machines and production lines.

\subsection{Levels of Breathing}

All life systems of the universe can be divided into four levels: 0 dimensional, 1 dimensional, 2 dimensional and 3 dimensional life [10]. Lives of different levels have different ways of breathing and communicate with the surroundings correspondingly. For example, the primitive society, the slave society, the feudal society, the capitalist society and the socialist society have their own different ways of breathing.

\section{Reproducing}

Reproducing is an important link of the four-seasonal movement of life. It is also life's important function.

\subsection{Reproducing of Living Beings}

When living beings develop to a certain stage of their four-seasonal development, they can then produce offspring similar to themselves. This is called reproduction. Humans have males and females. The reproductive cells of both sexes combine and produce offspring. Genetic information from both mother and father is brought to the next generation by the DNA in their reproductive cells. Reproduction is a way to carry on the family line and also one feature of the four-seasonal movement of living beings.

\subsection{Reproducing in the Broad Sense}

Life reproducing is to produce new individuals by male and female sexual intercourse. In the inorganic world, there are also male (state-varying organization [1]) and female (state-stabilizing organization [1]), which can also have sexual intercourse and produce new life. This is also reproduction. For example, life began on the earth when the male (state-varying organization [9]) and the female (state-stabilizing organization [9]) of the earth have intercourse. Man-made objects are reproductions of humans.

\subsection{Kinds of Living Being Reproducing}

According to Taoism and Buddhism researches, living beings have the reproducing ways of self-generation, generation by augmentation, and generation by transformation [11]. That is to say, different forms of life have different ways and results of reproducing.

\subsubsection{Self-Generation}

It refers to living beings producing individuals similar to father and mother by male and female 
pregnancy and intercourse. The reproducing of living beings belongs to self-generation. Self-generation consumes huge energy inside the body of father and mother and makes their body energy decrease.

\subsubsection{Generation by Augmentation}

It refers to living beings producing life energy to improve their internal energy and prolong their life span but not individuals similar to themselves or producing new life much smaller. The reproducing of the solar system and galaxies is of this kind. The male and female in the solar system and galaxies have intercourse and produce life energy. This energy provides lasting circle energy for the galaxies' own life movement and at the same time creates smaller lives on the earth, namely living beings. Generation by augmentation is good for a longer life span and living beings occurrence.

Likewise, when Taoists and Buddhist are practicing, they produce internal energy, which is good for a longer life span and better health, thus called generation by augmentation. Inside human (animals and plants) bodies, there are a large number of smaller living things: bacteria. Humans and animals nourish them. This is also called generation by augmentation.

\subsubsection{Generation by Transformation}

It is also called generation by creation. It creates from nothing. It is the combination of thoughts and life substances. Living entities produce through automatic organization of thinking. For example, the solar system, galaxies and the man-made world are all formed by automatic organization of thinking [2].

\section{Reproducing of the Solar System and Occurrence of Living Beings}

As has been instructed above, the reproduction of the solar system and galaxies is generation by augmentation, which is good for self improvement and life occurrence. Living beings are the result of celestial body generation by augmentation and the transformation of galaxy life (inorganic life) [1] into organic life (living beings).

\subsection{Reproducing Mechanism inside the Solar System}

Inside the solar system are male mechanism (state-varying organization) and female mechanism (state-stabilizing organization), both of which are interacting with each other (intercourse) all the time, producing seeds, namely seeds of life information. As the four-seasonal movement of the solar system produces new states all the time, reproduction of the solar system can produce different life information seeds.

\subsection{Four-Seasonal Ecosystem of Living Beings and the Ecological Universe}

Living beings on the earth form a gigantic ecosystem and regional areas form regional ecosystems too. All the ecosystems have four-seasonal structure and four-seasonal movement. This structure consists of the spring mechanism (inorganic surroundings), the summer mechanism (plants - energy producers), the autumn mechanism (consumers-first-level consumers, second-level consumers including animals and humans) and the winter mechanism (decomposer-bacteria) [6].

Living beings on the earth reflect life of the universe and are the reflection of celestial body life on the earth. Therefore, corresponding with the four-seasonal ecosystem of living beings, there is the ecological universe of four-seasonal movement [2].

Celestial bodies in the ecological universe have kinds of life information: gene information, thought information, energy information, structure information, etc. Living beings are breathing systems which exchange all sorts of information with the surroundings all the time. That is to say, living beings give out sorts of life information all the time. When life seed information of celestial bodies in the universe is released, it automatically flows to the reproduction location of the universe: the solar system and the earth.

\subsection{The Earth Is the Reproducing Location of the Solar System}

The earth is also a living being, which has male 
mechanism (state-varying organization-the atmosphere) and female mechanism (state-stabilizing organization - the solid layer) [1]. The two are having intercourse all the time and reproduction happens all the time. So the earth is the reproducing location of the solar system.

\subsection{Occurrence of Living Beings}

The internal interaction of the state-varying organization and state-stabilizing organization of the earth is also intercourse. Forces of the solar system and the earth interact based on the four-seasonal mechanism and produce all kinds of life substances that living beings need and the four-seasonal mechanism of living beings [9]. All the life information seeds of celestial bodies and the solar system gather on the earth and then combine automatically with all the life substances produced by the earth to produce living beings. This is how life occurs on the earth. Apparently, this kind of reproduction is generation by transformation. Living being reproduction afterwards is self-generation.

\section{Transformation of Different Life Forms}

Life forms in the universe can be divided into dominant lives (tangible lives): galaxies, living beings, society and the man-made worlds, and the recessive lives (intangible lives) that we cannot see [12], such as thoughts, spirits, ideas, etc. Different forms of life are transforming all the time.

\subsection{Transformation within Tangible Lives: Galaxies,} Living Beings, Society, Man-Made Worlds

Living beings in the universe (galaxies, living beings, society and man-made worlds) transform through different reproducing ways. That is: universe thoughts combine with universe life substances to organize and form automatically celestial bodies; thought information of celestial bodies combines with life substances in the solar system produced through generation by augmentation to organize and form automatically living beings; humans and animals automatically organize through thoughts to form society; human thoughts automatically organize to produce man-made worlds which return to nature after dying.

\subsection{Transformation between Tangible Lives and Intangible Lives}

Thoughts, spirits and ideas are all recessive or intangible lives. Tangible lives are the combination of thoughts with the human body. When tangible lives are controlled by genes, they will die after the four-seasonal cycle and thoughts separate from the human body, tangible lives transform into intangible lives, which are pure thoughts.

The universe is a living being where both tangible substances (life substances) and intangible lives (thoughts) are kept busy. Driven by life movement, the two are sure to combine to form tangible lives through generation by transformation, generation by augmentation and self generation. This is the transformation from intangible lives to tangible lives.

\section{Conclusion}

(1) The four-seasonal movement is the general movement of the universe. Galaxies, living beings, society, man-made worlds and all sorts of substances have or participate in the four-seasonal movement.

(2) The four-seasonal movement includes the transverse four-seasonal movement and longitudinal four-seasonal movement. The former is the breathing movement. All substances have breathing function. The mechanism of breathing is the result of interaction of repulsive force and attractive force. The latter is controlled by genes. The four-seasonal movement is confined. This is the reason that life has the function of automatic restoration. Life produces seeds and reproduction in the four-seasonal movement. The four-seasonal movement of life depends on the surroundings.

(3) Living beings are developed from seeds. All 
entities (celestial bodies, living beings, society and man-made objects) are expressions of thoughts, ideas, regulations, designs and methods. Thoughts, ideas, regulations, designs and methods are the seeds of celestial bodies, living beings, society and man-made objects.

(4) Breathing covers living being breathing, celestial body breathing, society breathing and man-made system breathing. Lives have four levels: 0 dimensional lives, 1 dimensional lives, 2 dimensional lives and 3 dimensional lives. Lives of different levels have different ways of breathing, communicating with the corresponding levels of the surroundings.

(5) Not only do living beings have reproducing function but also celestial bodies and society do. Ways of life reproducing mainly are: self-generation, generation by augmentation and generation by transformation. Living being reproduction belongs to self-generation; reproduction of the solar system and galaxies belongs to generation by augmentation; man-made objects are automatically produced by organizing human thoughts, thus called generation by transformation; society is also formed automatically through human organization; Galaxies are formed automatically through universe thought organization, called generation by transformation too.

(6) The solar system and the earth both have reproducing mechanism. The earth is the reproducing location of the solar system. The intercourse of the state-varying organization and state-stabilizing organization inside the earth produces all the life substances and the four-seasonal mechanism needed by life occurrence, based on the patterns of different forces and the four-seasonal mechanism of the solar system and the earth. All kinds of life information seeds of celestial bodies and the solar system gather on the earth and automatically combine with all kinds of life substances produced by the earth to produce living beings.

(7) Life forms in the universe include not only tangible lives (galaxies, living beings, society and man-made objects) but also intangible lives (thoughts, spirits, etc.). According to different reproducing ways of life, different forms of lives transform all the time.

\section{References}

[1] Yan, J. Z. 2013. "Introduction to Unity of Physics and Biology, Inorganic Life Materials." Journal of Physical Science and Application 3 (1): 58-64.

[2] Yan, J. Z. 2013. "Automatic Organization, Thinking Motion and Dynamics of the Universe." Journal of Physical Science and Application 3 (3): 199-208.

[3] Tian, H. L. 2006. The Original of the Book of Changes. Taiyuan: Shanxi Science and Technology Press, 12, pp244-55.

[4] Xie, H. 2011. The Illustration Canon of the Yellow Emperor. Beijing: TCM ancient books press.

[5] Yu, K. Z. 2013. Yin and Yang five Xings in mystery. Beijing: Huaxia Press.

[6] Yan, J. Z., and Yang, J. 2013. "Unity of physics and biology." Materials Protection 46 (S2): 169-71.

[7] Wang, X. G. 2012. The Magic of Human Life Rhythm. Beijing: Science and Technology Literature Press.

[8] Niu, X., and Zhang, Z. X. 2012. Physiology. Beijing: China press of Traditional Chinese Medicine.

[9] Yan, J. Z. 2014. "The First Push of the Universe and Origin of Life." Journal of Physical Science and Application 4 (3): 198-205.

[10] Yan, J. Z. 2014. "Beginning of a New Revolution of Science \& Technology." Journal of Physical Science and Application 4 (4): 269-75.

[11] Xiong, C. J. 2011. "Explication of Thought of Lao Zi's People Following the Erath.” Beijing: Tuanjin Press.

[12] Xiong, C. J. 2011. Tao Medicine. Beijing: Tuanjin Press. 